\title{
Dupilumab Injection in the Management of Steroid-Induced Rosacea: A New Case Report
}

\author{
Waqas S. Abdulwahhab ${ }^{1 *}$, Alaa S. Mehair ${ }^{2}$ \\ ${ }^{1}$ Consultant Dermatology \& Venereology/Department of Dermatology and Venereology, Al Qassimi Hospital, Sharjah, United \\ Arab Emirates \\ ${ }^{2}$ Department of Dermatology and Venereology, Al Qassimi Hospital, Sharjah, United Arab Emirates \\ Email: *wqs_saad@yahoo.com, alaa.alazawe@yahoo.com
}

How to cite this paper: Abdulwahhab, W.S. and Mehair, A.S. (2021) Dupilumab Injection in the Management of Steroid-Induced Rosacea: A New Case Report. Journal of Cosmetics, Dermatological Sciences and Applications, 11, 10-17.

https://doi.org/10.4236/jcdsa.2021.111002

Received: October 20, 2020

Accepted: March 5, 2021

Published: March 8, 2021

Copyright (c) 2021 by author(s) and Scientific Research Publishing Inc. This work is licensed under the Creative Commons Attribution International License (CC BY 4.0).

http://creativecommons.org/licenses/by/4.0/

\begin{abstract}
Background: Topical corticosteroids (TCS) are used frequently for various inflammatory skin conditions. The prolonged daily and inappropriate use of TCS may cause adverse effects such as erythema, atrophy, and telangiectasia. Steroid rosacea or addiction is a distinct adverse effect of inappropriate use of TCS. It occurs most commonly in adult women applying mid- or high-potency TCS to the face. Dupilumab is an interleukin 4 (IL-4) receptor $\alpha$-antagonist approved for treatment Moderate-to-Severe Atopic Dermatitis in childhoods and adults. The role of dupilumab injection in steroid-induced rosacea management is rarely discussed. Aim: To document a new case presentation of atopic dermatitis on high potency topical facial steroid addiction for a duration of 4 years in a young female patient who was successful treated with dupilumab injection and withdrawal therapy. Case Report: A 32-year-old female patient with a history of atopic dermatitis presented with a history of worsening pruritic facial rashes for a one-month duration and on high potency TCS (Clobetasol Propionate ointment $0.05 \%$ ) since 4 years ago with multiple treatments failure of steroidal withdrawal treated successfully in combination with dupilumab injection. Conclusion: Dupilumab injection considers effective and inducing rapid resolution of signs and symptoms of steroid-induced rosacea in patients with a history of atopic dermatitis in combination with withdrawal therapy without a rebound effect.
\end{abstract}

\section{Keywords}

Corticosteroid, Steroid Rosacea, Atopic Dermatitis, Dupilumab

\section{Introduction}

TCSs are used frequently for various inflammatory conditions in dermatology. It 
is well-known that the prolonged daily and inappropriate use of TCS may cause adverse effects such as atrophy, telangiectasia, and striae [1].

TCS withdrawal or steroid addiction occurs most common in adult women applying mid-or high-potency TCS to the face or genital region and is associated with increased frequency and duration of treatment [2] [3] [4].

This condition can be divided into 2 subtypes with distinct clinical presentations. The erythematoedematous variant is characterized by erythema, scaling, desquamation, and edema, with symptoms including burning, pruritus, pain, and decreased tolerance for emollients. The papulopustular type is distinguished by the prominence of pustules, papules, and nodules and the less common presence of edema and burning [5].

Although the mechanism of this phenomenon is not well understood, it is theorized to be caused by the effects of TCS on the local immune system and cutaneous blood vessels [6]. TCSs decrease the production of nitric oxide (NO), thereby inhibiting its vasodilatory action and depleting mast cells, which are regulated by NO [7] [8] [9]. In the absence of TCS use, a rebound effect may occur in which NO levels increase, leading to exaggerated vasodilation [6].

Steroid-induced rosacea is characterized by centrofacial, perioral, and periocular monomorphic inflammatory papules and pustules distributed in areas that have been chronically exposed to topical steroids, especially, of fluorinated type. The appearance is of a flaming red, scaly, and papule-covered face (red face syndrome) [10]. If left untreated, permanent skin atrophy and telangiectasia can result. Treatment involves discontinuation of steroids and administration of oral tetracycline or macrolides and non-steroidal topical preparations. Once therapy is begun, clearing of the lesions may take several months [11].

Dupilumab has recently been approved for the treatment of childhood and adult patients with moderate-to-severe atopic dermatitis [12]. It's an interleukin 4 (IL-4) receptor $\alpha$-antagonist that inhibits IL-4 and IL-13 signaling through blockade of the shared IL- $4 \alpha$ subunit. Blockade of IL- $4 / 13$ is effective in reducing Th2 response [13].

The combination of dupilumab injection and TCS withdrawal therapy is rarely discussed and the mechanism is not fully understood [14].

In the present case report, we are describing a young woman with a history of atopic dermatitis on high potency topical facial steroid addiction for a duration of 4 years who was successful treated with dupilumab injection and withdrawal therapy without rebound effect after 6 months follow up. The consent form was taken from the patient about the publication of her condition.

\section{Case Report}

A 32-year-old female patient presented to our clinic with a history of worsening pruritic facial rashes for the one-month duration. The patient's medical history was remarkable for allergic rhinitis and long-standing facial rash that appeared 4 years earlier diagnosed as atopic dermatitis for which she was on high potency 
TCS (Clobetasol Propionate ointment $0.05 \%$ ). The family history of atopy was positive for her mother. The patient had been using TCS over her face twice daily for the last 4 years, stopping only, at most, 3 or 4 days, as the rash would always return and became more worsening on discontinuation. The patient had a history of multiple treatments failure to steroid withdrawal, where aggravation of her facial rashes occur with more redness and burning sensation that cannot be tolerated when trying to tapering fluorinated topical steroid.

On examination, a young healthy woman, afebrile, with confluent erythematous edematous patches on the bilateral facial sides sparing periocular region were not applying steroid, associated with severe dryness, pigmentations, skin thinning, atrophy and telangiectasia with difficulty in moving her facial muscles expression and opening her mouth with prominent wrinkles, especially in the area above upper lip giving older age appearance (Figure 1).

The main differential diagnosis included steroid-induced rosacea and tinea incognito. No history of animal contact and $\mathrm{KOH}$ examination obtained and came up with a negative result. After discussion with the patient, the Planning for therapy recommendation was as follow:

- The topical Clobetasol Propionate ointment 0.05\% should discontinue. To minimize flare-up, slow withdrawal is obtaining by decreasing TCS applied and choosing another less potent type.

- Following up weekly for any evidence of flare-up.

- Starting dupilumab subcutaneous injection, $600 \mathrm{mg}$ loading dose, followed by $300 \mathrm{mg}$ every other week.

- Applying oil-free moisturizing agents.

Topical Betamethasone Dipropionate 0.05\% cream (class III) was started every other day for one week with instruction given to the patient to avoid sun exposure and using appropriate sunscreen.

After one week of therapy, the patient presented with a severe facial flare-up where numerous 2- to 3-ml pustules, papules, and nodules on erythematous base appeared, some coalescing, with hemorrhagic and yellowish dried crusts mostly distributed on the perioral area (Figure 2).

Doxycycline $100 \mathrm{mg}$ capsule was given twice daily. Discontinued Betamethasone Dipropionate $0.05 \%$ cream and started with topical Hydrocortisone 17Butyrate Lipo $0.1 \%$ cream (Class V) every other day for one week, Topical clindamycin lotion $1 \%$ daily and Tacrolimus (as monohydrate) ointment $0.03 \%$ every other day alternated with topical steroid. The second week after therapy, papules, nodules, and pustules started to resolve, reduced redness and pigmentation but more dried crusts persist mostly on the perioral area (Figure 3). Stopped Hydrocortisone 17-Butyrate Lipo cream $0.1 \%$ and prescribed Hydrocortisone acetate $1 \%$ cream (Class VII) every other day for one week. The third week after therapy, redness decreased and became discrete, pigmentation resolved, still few dispersed papules, nodules, and pustules (Figure 4). Hydrocortisone acetate $1 \%$ cream stopped and continued on the other medications. 
The fourth week after therapy, the patient presented with just slight erythema, completely resolved papules, nodules, and pustules, the disappearance of wrinkles, and no more dryness (Figure 5). We recommended continuing on the same medications for one month then discontinuing except topical moisturizer and dupilumab injection to avoid any possibility of relapse. 3 months later, there was complete facial clearing, the disappearance of erythema. Stopped dupilumab injection and continued on just moisturizer. For another 3 months followed-up, normal-appearing facial skin looks more glowing, younger, and no any signs of relapse (Figure 6).

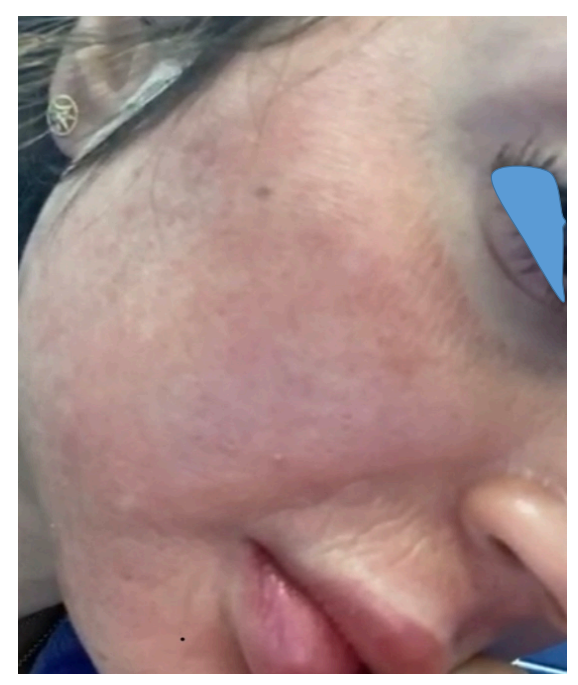

Figure 1. Steroid-induced rosacea, first visit, showed confluent erythematous edematous patches on the right side of the face sparing periocular region associated with dryness, pigmentation, skin thinning, atrophy, and telangiectasia. Note prominent facial wrinkles especially on the area above the upper lip giving the older age appearance of a 32 years old woman.

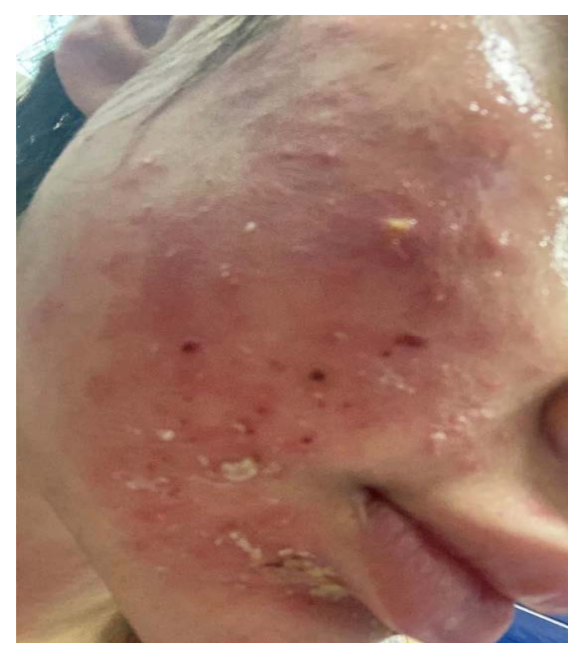

Figure 2. Steroid-induced rosacea, One week after therapy, showed numerous 2- to 3-ml pustules, papules, and nodules on an erythematous base, some coalescing, with hemorrhagic and dried crusts mostly distributed on the perioral area on the right side face of a 32 years old woman. 


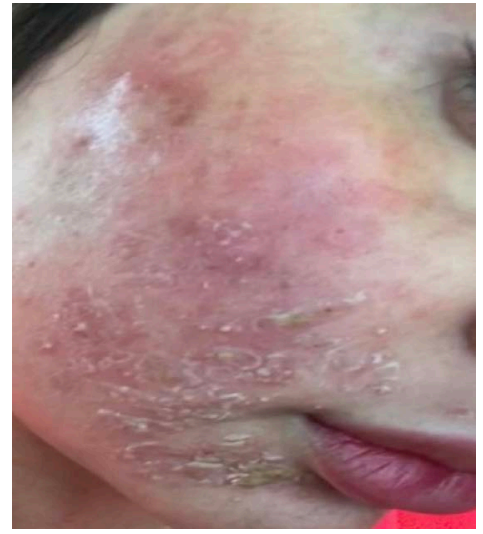

Figure 3. Steroid-induced rosacea, two weeks after therapy, showed resolved most papules, nodules, and some pustules, more dried yellowish crusts especially on perioral area, reduced redness and pigmentation on the right side face of a 32 years old woman.

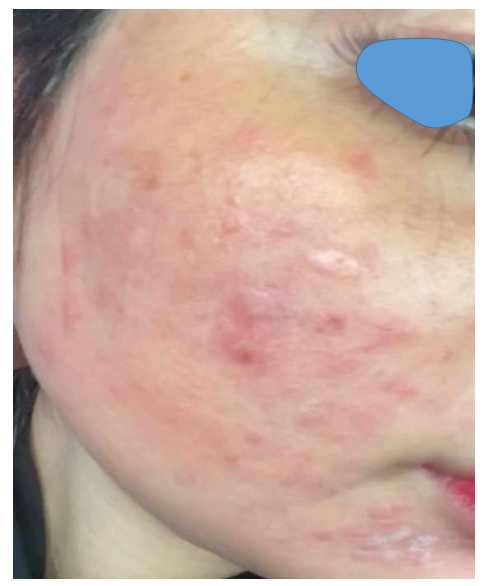

Figure 4. Steroid-induced rosacea, third week after therapy, showed more decrease in redness, resolved pigmentation, still few dispersed papules, nodules, and pustules on the right side face of a 32 years old woman.

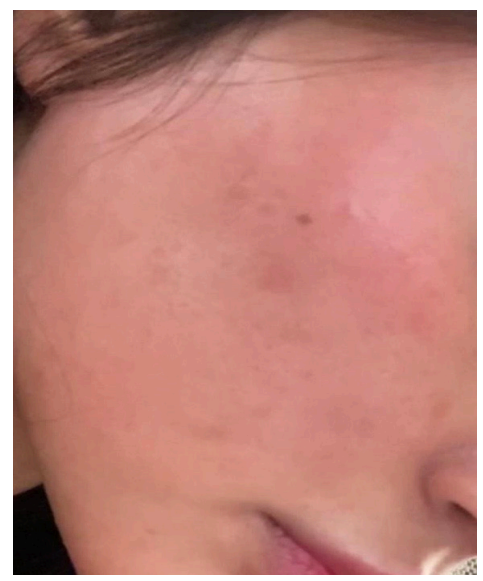

Figure 5. Steroid-induced rosacea, the fourth week after therapy, showed slight erythema, completely resolved papules, nodules, pustules, and no more dryness on the right side face of a 32 years old woman. Note complete disappearance of facial wrinkles on the area above the upper lip. 


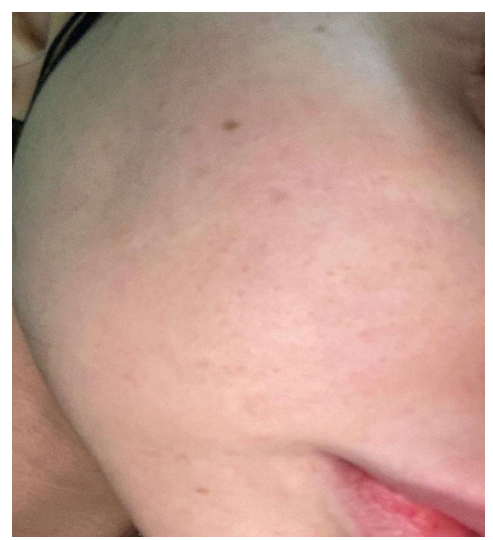

Figure 6. Steroid-induced rosacea, six months after therapy, showed normal-appearing facial skin, looks more glowing, younger without any signs for relapse on the right side face of a 32 years old woman.

\section{Discussion}

TCS withdrawal was first described by Sneddon in 1968 [15]. It is a clinical adverse effect of inappropriately prolonged and frequent use of mid-to high-potency TCS most commonly on the face or genital region [5].

It typically affects middle-aged women who have used a mid- or high-potency TCS on the face, usually for an indication of atopic dermatitis [2].

The role of dupilumab injection in combination with TCS withdrawal therapy in cases of steroid-induced rosacea is rarely discussed and the mechanism is not fully understood. The report done by Arnold et al. discussed 5 cases of atopic dermatitis started dupilumab injections several months after steroid withdrawal with worsening of their signs and symptoms before injection [14]. In our case presentation, we started dupilumab injection in combination with steroidal withdrawal therapy at the same time because of the patient's previous history of failure to withdrawal from therapy alone. The role of dupilumab injection in this combination might be enhancing the resolution of steroidal rosacea symptoms through its direct effect on the healing of underlying inflammation.

Dupilumab injection was safe in our case report without adverse effects [12] [13]. On the first visit, the patient presented with dominant erythematoedematous type of steroidal addiction and with started withdrawal, papulopustular type predominated as reported in the literature [5]. Steroid-induced rosacea is difficult to treat and maybe take several months for clearing [11]. The prolonged duration of therapy and patient non-compliance is the most common cause of treatment failure, but in our case, rapid resolution of signs and symptoms occurred with this combination within four weeks without relapse on followed up period for 6 months later.

In conclusion, dupilumab injection considers effective and inducing rapid resolution of signs and symptoms of steroid-induced rosacea in patients with a history of atopic dermatitis in combination with withdrawal therapy without a rebound effect. 
However, further studies are required with more reports to assess the role of this combination on the management of steroid-induced rosacea.

\section{Disclosure}

This study is an independent study and not funded by any of the drug companies.

\section{Conflicts of Interest}

The authors declare no conflicts of interest regarding the publication of this paper.

\section{References}

[1] Wolverton, S.E. (2012) Comprehensive Dermatologic Drug Therapy. 3rd Edition, Saunders Elsevier, Philadelphia, 487-504.

[2] Dhossche, J., Simpson, E. and Hajar, T. (2017) Topical Corticosteroid Withdrawal in a Pediatric Patient. The Journal of the American Academy of Dermatology Case Reports, 3, 420-421. https://doi.org/10.1016/j.jdcr.2017.06.006

[3] Fukaya, M., Sato, K., Sato, M., et al. (2014) Topical Steroid Addiction in Atopic Dermatitis. Drug, Healthcare and Patient Safety, 6, 131-138. https://doi.org/10.2147/DHPS.S69201

[4] Sheary, B. (2016) Topical Corticosteroid Addiction and Withdrawal-An Overview for GPs. Australian Family Physician, 45, 386-388.

[5] Hajar, T., Leshem, Y.A., Hanifin, J.M., et al. (2015) A Systematic Review of Topical Corticosteroid Withdrawal ("Steroid Addiction") in Patients with Atopic Dermatitis and Other Dermatoses. Journal of the American Academy of Dermatology, 72, 541-549. https://doi.org/10.1016/j.jaad.2014.11.024

[6] Rapaport, M.J. and Lebwohl, M. (2003) Corticosteroid Addiction and Withdrawal in the Atopic: The Red Burning Skin Syndrome. Clinics in Dermatology, 21, 201-214. https://doi.org/10.1016/S0738-081X(02)00365-6

[7] Di Rosa, M., Radomski, M., Carnuccio, R. and Moncada, S. (1990) Glucocorticoids Inhibit the Induction of Nitric Oxide Synthase in Macrophages. Biochemical and Biophysical Research Communications, 172, 1246-1252. https://doi.org/10.1016/0006-291X(90)91583-E

[8] Lavker, R.M. and Schechter, N.M. (1985) Cutaneous Mast Cell Depletion Results from Topical Corticosteroid Usage. The Journal of Immunology, 135, 2368-2373.

[9] Coleman, J.W. (2002) Nitric Oxide: A Regulator of Mast Cell Activation and Mast Cell-Mediated Inflammation. Clinical and Experimental Immunology, 129, 4-10. https://doi.org/10.1046/j.1365-2249.2002.01918.x

[10] Goldman, D. (2001) Tacrolimus Ointment for the Treatment of Steroid-Induced Rosacea. A Preliminary Report. Journal of the American Academy of Dermatology, 44, 995-998. https://doi.org/10.1067/mjd.2001.114739

[11] Bhat, Y.J., Manzoor, S. and Qayoom, S. (2011) Steroid-Induced Rosacea: A Clinical Study of 200 Patients. Indian Journal of Dermatology, 56, 30-32. https://doi.org/10.4103/0019-5154.77547

[12] Igelman, S., Kurta, A.O., Sheikh, U., McWilliams, A., Armbrecht, E., Jackson Cullison, S.R., Siegfried, E., et al. (2020) Off-Label Use of Dupilumab for Pediatric Patients with Atopic Dermatitis: A Multicenter Retrospective Review. Journal of the 
American Academy of Dermatology, 82, 407-411.

https://doi.org/10.1016/j.jaad.2019.10.010

[13] Gooderham, M.J., Hong, H.C., Eshtiaghi, P. and Papp, K.A. (2018) Dupilumab: A Review of Its Use in the Treatment of Atopic Dermatitis. Journal of the American Academy of Dermatology, 78, S28-S36. https://doi.org/10.1016/j.jaad.2017.12.022

[14] Arnold, K.A., Treister, A.D. and Lio, P.A. (2018) Dupilumab in the Management of Topical Corticosteroid Withdrawal in Atopic Dermatitis: A Retrospective Case Series. JAAD Case Reports, 4, 860-862. https://doi.org/10.1016/j.jdcr.2018.06.012

[15] Sneddon, I. (1968) Varicose Ulcers and Use of Topical Corticosteroids. British Medical Journal, 1, 579-580. https://doi.org/10.1136/bmj.1.5591.579-c 